\title{
Colloidal suspensions, Brownian motion, molecular reality: a short history
}

\author{
M D Haw \\ Department of Physics and Astronomy, University of Edinburgh, King's Buildings, \\ Mayfield Road, Edinburgh EH9 3JZ, UK \\ E-mail: M.Haw@ed.ac.uk
}

Received 25 June 2002

Published 9 August 2002

Online at stacks.iop.org/JPhysCM/14/7769

\begin{abstract}
In the first decade of the 20th century, nearly a hundred years of work on the phenomenon of Brownian motion culminated in theory and experiments that demonstrated irrefutably the discontinuous or molecular nature of matter. Colloidal suspensions and the phenomenon of Brownian motion thus became the key to confirmation of the 'new world-view' of statistical mechanics, the statistical basis of thermodynamics. One may conveniently identify four 'stages' in the historical development of 'Brownian motion science', characterized in turn by discovery, observation, theoretical prediction, and quantitative confirmation. We are living, of course, in the fifth stage, that of application: the modern discipline of 'soft matter'.
\end{abstract}

\section{Introduction}

To study colloidal suspensions is in many respects to study the consequences of Brownian motion. It is easy for anyone working in experimental colloid science nowadays to become rather blasé about Brownian motion - as seen straightforwardly in the optical microscope or in the fluctuating speckle pattern produced by the scattering of a laser beam through a colloidal suspension. But of course the phenomenon of Brownian motion, whose first recorded observations date back to the early part of the 19th century, actually held the key to some of the great achievements of modern condensed matter physics: the establishment of statistical mechanics as an immensely successful 'world-view'; the confirmation of the reality of the molecular nature of matter; and the demonstration of the importance of thermal forces in the physics of 'everyday' phenomena. The study of colloidal suspensions has thus provided us with a fundamental picture of reality — reality on scales that go far beyond the two or three orders of magnitude of the size range of the humble colloidal particle.

In this paper I give a brief historical account of the development of the science of 'Brownian motion', up to and including the theoretical work and experimental studies that combined to establish, nearly a hundred years ago, the reality of the molecular-kinetic view of matter. 
In a concluding section I very briefly consider the status of colloidal science immediately following Jean Perrin's experimental 'confirmation' of molecular reality, and the importance of this 'classical' verification of the statistical method to the subsequent rise of quantum mechanics.

The story of Brownian motion is one of confused experiment, heated philosophy, belated theory, and, finally, precise and decisive measurement. This paper must necessarily be brief; for more detail, the best sources are undoubtedly the original publications, particularly those of Gouy, Einstein, Langevin, and Perrin; the work of Perrin, put into its historical context, is extensively described in Nye's book [1].

\section{Brownian motion observed: 1827-1905}

\subsection{Discovery}

That the name of botanist Robert Brown should be singly associated with the phenomenon of 'Brownian motion' is perhaps somewhat fortuitous and not entirely justified ${ }^{1}$. It seems that the ubiquitous, irregular motion of small grains suspended in fluids had been observed very soon after the appearance of the microscope [3]. Generally, these early observations were interpreted as the motion of living creatures ( 'animalcules'). Brown's first contribution was to carry out, 'in the months of June, July and August, 1827', detailed observations of the motion of pollen grains suspended in water [4]. In fact similar observations had been recorded in France, in 1827, by Adolphe Brongniart [5], while Brown's account did not appear until a year later. Both Brongniart and Brown at first presumed a 'living' origin of the motions, but Brown subsequently showed that inorganic grains also demonstrated an irregular motion in suspension. Observations were also made of gas bubbles in liquid inclusions trapped inside mineral crystals. That these irregular, apparently inexhaustible motions were observed in such a wide range of suspensions put paid to the idea of a 'vital' cause, demonstrating that the explanation, whatever it was, lay more in the realm of physics than biology.

\subsection{Observation}

Subsequent to its 'discovery', the story of 'Brownian motion' (as the irregular motion became known) was confused by many conflicting reports as to the influence of external factors, such as vibrations, microscopic currents, thermal variations set up by the illuminating light, capillarity, and so on. Many physical hypotheses and explanations of Brownian motion were put forward, including electrical effects, polarity, surface tension, temperature gradients, and even 'radiating caloric' (see [1, p 12]). The important question, still causing controversy as late as the end of the century, was whether the observed motion could indeed be entirely explained by external effects. As late as 1898, 70 years after Brown and Brongniart, it was still being claimed that the motion was due to 'temperature differences' between the liquid and the surroundings (see [1, p 23] for a detailed discussion). Solvent effects, such as coagulation of the particles induced by addition of acids, also confused the issue.

Brongniart himself was satisfied almost from the start that external causes could not explain Brownian motion; his opinion was not, however, universally shared. Until scientists could be convinced that Brownian motion was a fundamental physical property of fluids, many would be reluctant to look at the phenomenon very closely. Its possible significance to thermodynamics thus went largely unappreciated until the last decade or so of the century.

1 This is perhaps an instance of what is apparently known as 'Stigler's law of eponymy'; see e.g. [2]. 
The publications of Leon Gouy, in the late 1880s, mark an important step in the struggle to establish a solid basis of experimental fact, from which scientists could begin to consider the fundamental implications of Brownian motion. In a series of comprehensive experiments [6-8] Gouy demonstrated convincingly that the irregular motion of suspended particles was not a result of external vibration, temperature, incident light, surface tension, etc: in other words that Brownian motion was indeed a fundamental physical property of fluid matter. Gouy verified previous reports of qualitative trends, such as the decrease of 'vigour' of the observed motion with increasing particle size and increasing solvent viscosity. Perhaps most importantly, Gouy also pointed out [7] the significance of Brownian motion as regarded 'Carnot's principle' (i.e. the second law) in thermodynamics. Gouy proposed a thought experiment in which a 'paddle' attached to a tiny thread would be driven back and forth continually due to the impacts of suspended Brownian particles. The thread, emerging from the suspension, might be used to drive a wheel: this would look suspiciously like the supposedly illegal 'perpetual motion'. In effect Gouy was proposing that the second law could not be considered 'absolute' at microscopic scales; in other words that thermodynamics required a statistical interpretation in which its absolute character only became visible in what we would now call the 'thermodynamic limit' of large numbers. Such an idea had of course already been introduced hypothetically, in the kinetic theory by (amongst others) Clausius, Boltzmann, and Maxwell, and in thermodynamics through Maxwell's demonic speculations: but Brownian motion, argued Gouy, demonstrated the statistical nature of the laws of thermodynamics in reality.

However, it would be simplistic to suppose that the comprehensive work of Gouy finally silenced the various conflicting ideas surrounding Brownian motion and its relation to kinetic theory. As pointed out by Nye [1, p 21], while Gouy was discussing Brownian motion as a demonstration of the molecular-kinetic theory 'in the flesh' [8], in the very same 1895 volume of the Revue Générale des Sciences, Ostwald was berating the atomists for following a hypothesis that could never be experimentally verified [9] $]^{2}$. In fact, Brownian motion, more than half a century after Brown's and Brongniart's first reports, now found itself at the heart of a deep rift in the philosophy of science.

\section{Brownian motion and philosophical conflict: late 19th century}

The latter part of the 19th century was an epoch of doubt and controversy amongst physicists, physical chemists, and philosophers of science. The 'continuous' description of reality, as demonstrated for instance in differential field descriptions such as the electromagnetic theory of Maxwell, vied against the 'discontinuous' hypothesis most clearly advanced in the kinetic theory of Boltzmann. The success of Maxwell's theory led many to hope that all natural phenomena might be describable in terms of continuous differential equations; as had been the dream since Newton's successful calculus of gravity in the Principia.

Meanwhile, underneath the particular continuum-discontinuum debate, there lay the more general question of hypothesis versus description. In the spirit of description, Newton had developed and validated a mathematical description of gravitation, but had explicitly avoided any attempt to explain the origin of the forces ${ }^{3}$. What was the good of a hypothesis such as the molecular-kinetic theory, argued the 'continuists', when it was unlikely ever to be directly verifiable? The laws of thermodynamics, developed and refined following observation and experiment, successfully described many processes; an underlying 'hypothesis' such as

2 Wilhelm Ostwald was well known for his insistence on the importance of direct experimental evidence, and it is unclear whether he knew at this time of Gouy's work; after the later, quantitative work of Perrin, Ostwald was duly converted to 'atomism' as an actual description of reality.

3 Interestingly, in the Opticks, Newton did go further, in speculating on the particulate nature of light. 
kinetic theory was unnecessary. A hypothesis or analogy might have some value while it was being used to arrive at correct forms for 'laws' (e.g. Maxwell's mechanistic picture of electrodynamics); after that it ought to be abandoned. Molecules, said the continuists, were a convenient fiction, but scientists should beware of being seduced into belief. There was no sense wasting time investigating the fine points of a hypothesis that was not necessary to describe the 'laws' of reality.

There was also, perhaps, a political danger in allowing the tenets of thermodynamics to be downgraded from solid laws to ephemeral averages. Around the end of the 19th century, especially in France (where a little later much of the important experimental and theoretical work on Brownian motion would be done), there was a growing popular and political despair over the apparent failure of the sciences to grasp reality. Perhaps science had oversold itself in the past; many intellectuals now doubted that the scientific viewpoint would prove to be of any lasting use at all. The apparently statistical slant given the Second Law by kinetic theory worried many scientists who had hoped to build a complete theory of matter based on the 'energetics' of the laws of thermodynamics. Should even thermodynamics be reduced to the status of probability, might not the whole edifice finally crumble?

The 'discontinuists', meanwhile, were adamant that the hypothesis of the kinetic theory could be viewed as an actual description of reality [8]. Most of the discontinuists were also convinced that science could not be done without 'hypothesis'; that a purely superficial description of phenomena was not enough. What the discontinuists needed was a direct demonstration of the discontinuity of matter. Brownian motion was recognized by scientists such as Gouy, and later Jean Perrin, as the best candidate for just such a 'reality check'.

\section{Theory of Brownian motion: 1905-1908}

Early attempts to quantify anything in Brownian motion had met with great difficulties (see below). In fact, before the parallel theoretical treatments of Einstein, Smoluchowski, and Langevin, the real problem was that the experimentalists did not know quite what to measure. But after 1905, theory, even when incompletely comprehended, provided a framework that guided the experimentalists toward the most meaningful measurements.

In the opening paragraphs of his first 'Brownian motion' paper of $1905[10]^{4}$, Einstein was in fact rather coy about explicitly connecting his theoretical ideas with Brownian motion as observed in colloidal suspensions. However, it is clear that Einstein was fully aware of the importance that an experimental confirmation (or refutation) of his results would have, as regarded the reality of the molecular-kinetic theory. The somewhat 'roundabout' route taken by the 1905 paper seems expressly designed to demonstrate the vital contradiction between this theory and classical thermodynamics.

Thus Einstein began the 1905 paper with a demonstration that, should colloidal particles be considered (as required by the molecular-kinetic theory) as essentially very large molecules, there must exist a 'colloidal osmotic pressure'. If a partition were placed in a suspension so as to restrict the particles to one half, the particles must exert a force on the partition (just as molecules would in a gas, leading to the familiar 'gas' osmotic pressure). Classical thermodynamics implied, however, that the free energy of the particles could not depend on the position of the partition but only on the number of particles; and therefore, classically, there could be no osmotic force.

By supposing an equilibrium between an (imaginary) force imposed on a particle and the 'restoring' force of the 'colloidal' osmotic pressure, Einstein then went on to obtain an

4 English translations of Einstein's major Brownian motion papers may be found in [11]. 
expression for the 'diffusion constant' of a suspended particle. (A real example of such a situation is the sedimentation equilibrium of a suspension in a gravitational field: as would be measured later by Perrin.) Supposing, in dynamic equilibrium, on the one hand that the colloidal osmotic pressure balances the applied force, and on the other hand that the Stokesian particle flux produced by the applied force is cancelled by a diffusive 'current' of particles, Einstein thus obtained an expression for the 'diffusion coefficient' characterizing such a current, in terms of the colloidal osmotic pressure. Assuming a Stokesian form for the particle velocities imposed by the applied force, an expression for the diffusion coefficient is thus obtained, containing Avogadro's number $N$, the absolute temperature, the particle size, and the solvent viscosity.

The problem now was to predict the time dependence of the motion of a particle (which could be observed in an experiment) in terms of the above-derived diffusion coefficient. Einstein turned to a probabilistic consideration of the diffusive motion, in particular to obtain the mean displacement of a particle from some origin in terms of time. The appeal to (mean square) displacements was a key step: attempts to measure velocities of Brownian particles in experiments had failed because these velocities were too high and changed too rapidly. Indeed experiments had demonstrated the apparently bizarre result that the measured velocities (displacements divided by observation time) simply increased, apparently without limit, as the measuring time was reduced. The problem, of course, was the non-linearity of displacement with time. Einstein obtained the now-familiar expression showing that the mean displacement increased with the square root of time; and into this expression he was able to insert his relation for the diffusion coefficient. Concluding the 1905 paper, Einstein gave an estimate for the magnitude of displacement expected given typical particles in water, assuming a value for Avogadro's number, $N$. He also pointed out that, conversely, a measurement of the displacement ought actually to provide a measured value for $N$.

It is perhaps interesting to note in passing that a probabilistic analysis equivalent to Einstein's had actually already been applied, not to colloidal diffusion but to the fluctuation of share prices on the French stock market. In a paper published in 1900 [12], Bachelier arrived at the same 'displacement' law, this time for the 'mean displacement' of stock prices over time. This was perhaps the earliest example of the application of statistical mechanics to the 'science' of finance.

Meanwhile Marian von Smoluchowski, who had begun work on the problem of Brownian motion around 1900 but did not publish until 1906 [13], obtained essentially the same expression for the time dependence of displacement as Einstein, though with a numerically different coefficient (later found to be in error by Langevin). Importantly, Smoluchowski countered one argument against the molecular origin of the irregular motion of large particles, which was that any molecular 'impulse' could never give a strong enough push to generate the observed displacements. The error lay basically in assuming that a visible displacement (a substantial fraction of a micron, say) must arise from a single 'push'. Unlike Einstein in his original paper, Smoluchowski also considered the experimental literature, making a clear unhesitating link between the diffusion theory and Brownian motion. Finally, Smoluchowski obtained an expression that directly predicted an exponential 'sedimentation equilibrium' equivalent to the 'aerostatic' law describing the variation of atmospheric pressure with height: the same sedimentation equilibrium measured later in the first experiments of Jean Perrin.

Two years after Smoluchowski's first publication, Paul Langevin demonstrated yet a third derivation of the time dependence of the diffusion coefficient [14]. Langevin's derivation, however, was spectacularly simple and direct compared to the others (this is probably why it is Langevin's derivation rather than Einstein's that is usually found in modern textbooks). Langevin simply equated forces on the colloidal particle, directly introducing a 
fluctuating random force to represent the impulses of the molecules, and using, as Einstein and Smoluchowski had done, a Stokesian description of the 'opposing' frictional force due to the motion of the particle through the liquid. Through the theorem of the equipartition of kinetic energy (which is essentially where kinetic theory enters the argument) the mean particle velocity was linked to the temperature and to Avogadro's number. Langevin obtained an expression consisting essentially of Einstein's diffusion term, plus a decaying exponential correction. This last term resolved what had been an assumption in Einstein's method, that beyond some 'Brownian time' (the characteristic decay time in the exponential term) the particle motion could be assumed completely 'diffusive'. Langevin concluded his paper with an appeal for more accurate measurements, pointing out the importance of the use of 'grains of dimensions more easily and exactly known, and for which the application of Stokes's formula ... is definitely more legitimate'. It was Langevin's friend, Jean Perrin, who would finally provide the determining measurements.

\section{Quantitative measures from Brownian motion: 1906 onwards}

\subsection{First attempts}

In the following years a number of experimentalists took up the search for confirmation (or refutation) of these first theories. In 1906 Theodor Svedberg published measurements that he claimed were in agreement with Einstein's formula [15], though Einstein himself (and Jean Perrin) had difficulties with Svedberg's results and with his 'oscillatory' concept of Brownian motion (see [1, p 127]). Hopes for confirmation of Einstein's formula for the diffusion constant seemed to be further dented by the experiments of Victor Henri, published in 1908 [16]. In a pioneering use of a film camera (moving pictures had been invented not much more than a decade before) connected to a microscope, Henri was able directly to record the trajectories of latex particles in a suspension. Henri, while confirming to a reasonable degree Einstein's prediction of the scaling of the mean displacement with time, did not however find quantitative agreement with Einstein's formula for the diffusion coefficient. In fact the reasons for this remain unclear even now; Perrin, in 1909, was forced to put the disagreement down to unknown experimental problems [17]. (Aimé Cotton suggested that the intense illumination required to make the movies may have affected the suspension [18].) Henri himself concluded that there was some doubt about Einstein's results, suggesting that perhaps the use of the Stokes formula for the velocity of particles under the applied force was not correct, given the size of the particles. (A modern advance on the methods of Henri, using all the paraphernalia of confocal imaging, digital image processing, and so on, appears in the recent studies of diffusion in colloidal glasses by Weeks et al [19]. After nearly a hundred years of research, quantitative colloidal microscopy has progressed from a concentration of $\Phi \approx 0.001$ to 0.58 !.)

\subsection{Success through determination: Jean Perrin}

It fell to Jean Perrin and his team of students at the Sorbonne in Paris, in a set of papers beginning in 1908, to provide the tour de force of precision and sheer patient measurement which finally confirmed the theorists' predictions and settled the molecular-kinetic theory on a firm observational basis. In 1926 Perrin received the Nobel prize for this work (in the same year that Svedberg received the Chemistry prize for 'dispersion studies' and more particularly for the invention of the 'ultracentrifuge'). Perrin was a keen publicizer of science, both amongst his fellow scientists and at more public and political level: he was instrumental, later on, in the establishment of the French CNRS and the 'Palais de la Découverte' in Paris. His outspoken 
anti-Fascism made it necessary for him to flee France in 1940; he died two years later in the USA, with his country still under Nazi control.

In carrying out his first experiments, Perrin remained unaware of the details of Einstein's and Smoluchowski's theoretical work (though he knew of Einstein's theory, as demonstrated in a closing comment to Perrin's first paper of 1908 [20]). According to Nye [1, p 87] this was a normal method of work for Perrin: he would make only the briefest survey of the literature before embarking on experiments. On the other hand, Perrin was a close friend of Langevin, and was undoubtedly guided by Langevin as to his experimental strategy. As his results collected, of course, Perrin made a more comprehensive study of the theoretical background, aware that it was within the scope of his experiments to make a very strong case for the molecular-kinetic theory.

The value of the precision of Perrin's experiments cannot be underestimated. Confirmation of Einstein's theory required great precision in measurement of the size of particles, great control over their size distribution, and a high level of statistical accuracy. Doubtful measures of particle size and velocity had already been pointed out as a flaw in Theodor Svedberg's earlier work. Perrin described his (and his students') efforts to attain maximum precision in detail in his 'summary' paper of 1909 [17]. Firstly, to measure particle size, Perrin developed methods whereby a countable number of particles might be deposited on a surface and photographed: size could then be determined by measuring strings of touching particles. Perrin also used Stokes's formula to measure particle sizes via sedimentation velocities. In a third approach, Perrin developed a method of actually counting the total number of particles in a known volume of suspension; combined with the known mass and density of the suspension, this allowed calculation of the particle radius. By making the solvent very slightly acidic, Perrin found that he could force the particles to adhere to the glass walls of the microscope cell, and thence count their number. For the 'best' experimental samples (i.e. those with the narrowest size distribution) all three methods gave radii that agreed within ${ }^{5}<1 \%$.

To obtain as narrow a size distribution as possible, Perrin carried out an exhaustive series of 'size fractionations' by centrifugation. In a typical case, from $1200 \mathrm{~g}$ of starting material he might extract a few tenths of a gram of particles considered suitably 'monodisperse' for experiments. Such careful 'fractionation' was one of the keys to Perrin's success.

The choice of material was important, since all theories at this stage assumed a 'billiardball' (purely hard repulsive) interaction, between spherical particles. Perrin favoured particles of gamboge ('gomme gutte' in French), a resin derived from the sap of the tree Garcinia xanthochymus. Gamboge was sometimes used as a pigment by oil-painters: traces have been discovered in the paintings of Rembrandt and in the paint-box of J M W Turner. From Perrin's point of view the importance of gamboge lay in that it is easily dispersed in water, and that once dispersed it forms an exceptionally stable suspension of spherical particles that neither coagulate with each other nor (under normal conditions) adhere to the glass walls of the microscope cell. Gamboge had been used before, particularly by Gouy (with whose publications Perrin certainly was familiar from the beginning [20]). In follow-up experiments, Perrin's students also used particles of 'mastic', in order to show that the results did not depend on the material-an important argument for the 'universality' of the confirmation of Einstein's theory.

But perhaps the overriding element in the strategy of Perrin and his students was a thoroughgoing patience: after the exhaustive preparation of samples via repeat fractionations, extensive series of experiments, involving counting many thousands of particles, made true statistical precision possible. After the initial publications of 1908 [20,21] and the 'summary'

5 This agreement, as Perrin noted, incidentally provided evidence that the Stokes formula did apply accurately at the colloidal scale. 
of 1909 [17], Perrin continued to refine his results and methods [22, 23], determined that he would not leave any crevice into which the old 'continuists' might later on reinsert some wedge of doubt. Full details of Perrin's and his students' experiments may be found in Nye [1] and, indeed, are described by Perrin himself in his book Atoms [24]. For reasons of brevity, we consider here, as an example, only Perrin's earliest experiments, on sedimentation equilibrium distributions in colloidal suspensions. These experiments were an inspired starting point for Perrin, in that they avoided Svedberg's and Henri's problems with the direct measurement of displacements or velocities, yet still went to the heart of the question: the applicability, or not, of the molecular-kinetic theory to a real, observable system.

\subsection{Sedimentation equilibrium}

Difficulties always seemed to arise in the direct measurement of Brownian particle velocities or trajectories; as already mentioned, measurements of velocity via displacement versus time had been shown to give continuously increasing velocities as time intervals were reduced. According to Perrin's report of 1909 [17], he himself first tried such direct measurements of displacement and velocity, without success. Subsequently, he turned instead to measurements of the sedimentation equilibrium in a colloidal suspension. While not directly confirming the diffusion relation, such a measurement could give an estimate of the mean energy of a colloidal particle, thus validating or refuting the use, for colloidal particles, of the equipartition of energy in the kinetic theory, which predicted that the colloidal particle should have the same mean energy as a molecule. Measurement of sedimentation equilibrium could also provide an estimate of Avogadro's number, which, when compared with estimates from studies of gases, would further indicate whether Brownian motion might indeed be explained by molecular motion consistent with kinetic theory. Of course, Einstein's original development itself proposed an equilibrium between imposed forces and osmotic pressure in a suspension; though it seems that it had not occurred to Einstein himself that a measurement of sedimentation equilibrium would present a confirmation of the theory much more straightforward than direct measures of diffusion.

Perrin's first experimental paper of 1908 [20] gives a beautifully clear description of his first sedimentation experiments, and their analysis which explained the observed exponential distribution of particle concentration with height in the suspension. To measure the distribution, Perrin observed samples of gamboge suspension, sealed in a cell beneath a microscope cover slide, using a microscope with a narrow depth of field (ensuring the precise 'selection' of sharply imaged particles at a given height in the suspension). Before any measurements were taken, samples were allowed to 'equilibrate' over some time; measurements were also repeated after longer time intervals, to check that the suspensions were indeed in 'sedimentation equilibrium'. Perrin counted the number of particles at a series of heights (varying height simply by moving the objective lens up and down). Because of the difficulty of accurately counting a large number of particles in the visual field at one 'instant', in the first experiments Perrin actually used a very small image area which would typically contain only five or so particles: these might be 'counted' instantaneously, in effectively one 'glance' at the image. At a given height the numbers of particles in multiple 'glances' over a certain time interval were recorded, hence allowing an average relative particle concentration with height to be measured. In later experiments larger visual fields were recorded 'instantaneously' in photographic images, from which sharply focused particles could be identified and counted.

The results revealed that the number concentration of particles varied exponentially with height (in the case of the gamboge particles, being more dense than the suspending water, the concentration of course decreased with increasing height). For a stable concentration 
distribution $n(h)$ the gravitational force on the particles in a given horizontal 'slice' of the suspension, of thickness $d h$ at height $h$, is balanced by the difference between the colloidal osmotic pressures on the two faces of the slice. The osmotic pressures, according to the kinetic theory, are proportional to the particle concentrations $n$. Hence Perrin equated these forces in an equation involving the number concentration of particles and the thickness of the 'slice'. Integrating, one obtains the measured exponential distribution of concentration with height. As Perrin points out, the colloidal suspension is hence just like the atmosphere; with the difference that, across a depth of suspension of one tenth of a millimetre, one finds a change in concentration equal to that which would be observed in $6 \mathrm{~km}$ of the atmosphere.

Moreover, since Avogadro's number $N$ appears in the relation between colloidal osmotic pressure and concentration, one can use the measurement of $n(h)$ to estimate $N$. Alternatively, taking an assumed value for $N$ (at the time the 'best' value was around $7 \times 10^{23}$ ), one may compare the mean kinetic energy of a particle with that expected for a gas molecule at the same concentration $(=n R T / N)$. Hence, in Perrin's words, his measurements showed that 'the mean kinetic energy of a colloidal granule is thus equal to that of a molecule'. He goes on: 'This is, established by experiment,... the theorem of the equipartition of kinetic energies. At the same stroke, the kinetic theory of fluids appears rather fortified, and molecules a little more tangible'.

Building on these first measurements, as already described, Perrin and his students went on to construct a numerical mountain of experimental data [20-23], all of which gave results consistent with each other and with, for instance, other measures of $N$ and with the molecularkinetic theory. He and his students also perfected direct measurements of displacements (which had presented Henri and Svedberg with such difficulty) to confirm Einstein's diffusion theory. Experiments at a range of solvent viscosities, with a range of different colloidal materials, and under various external conditions, all combined irrefutably to convert the molecular-kinetic theory from a hypothesis to a description of matter. Through Perrin's and his students' efforts, the colloidal suspension had demonstrated the molecular nature of reality.

\section{Colloidal suspensions: from centre stage back to the wings}

The 'energeticists' at the turn of the 20th century were disappointed by and often hostile to Boltzmann's statistical interpretation of thermodynamics. If science is the search for the fundamental 'mechanism' of reality, then the replacement of the 'laws' of classical thermodynamics with the statistics of the molecular-kinetic theory must have seemed something of a setback, at least to those who believed that in the absolute thermodynamic laws the fundamental mechanism had at last been glimpsed. Perrin's confirmation of the reality of the statistical kinetic 'hypothesis' required then that the search for the 'fundamental' go even deeper. If the behaviour of matter at the super-molecular scale was dominated by statistics, one must go to still smaller scale to identify what it was that the counting statistics of kinetic theory actually counted.

Hence the energeticists' search for the fundamental mechanism had not been abandoned entirely; but rather, seeing that classical thermodynamics was after all a matter of statistics, the search for the fundamental plunged deeper. Surely if classical, 'mesoscale' thermodynamics was only a question of counting, at the next level down some solidity might yet be recovered: some single object, the counting up of which led to the statistics of colloids and Brownian motion, but whose individual behaviour might be found to follow some definite non-statistical 'laws'.

This continuing desire for something non-statistical and fundamental was perhaps one factor in the subsequent rapid rise, in the second and third decades of the 20th century, of the 
atomic, the subatomic, and the quantum as the 'cutting edge' of physics. Having barely left time for the consequences of the classical molecular-kinetic theory to be digested, physicists pushed breathlessly onward, still in search of the elusive solidity of a non-statistical 'law' or 'reality'.

So the colloidal suspension, having just reached the height of its stage career, found itself quickly relegated again to playing bit parts. Especially through the work of Einstein, the establishment of statistical thermodynamics coincided closely with the new developments of quantum mechanics. The radiation studies of Planck also seemed likely to give an even better measure of Avogadro's number. And at the same time, in the realm of experiment, the atom itself was being probed more and more deeply, by experimentalists such as Rutherford. While it is perhaps somewhat unfair, it is also perhaps understandable, that the colloid, which had just done such sterling work for Perrin, so abruptly lost its sex-appeal compared to the atom and the quantum.

Nevertheless, another defeat, and perhaps a greater one, awaited physicists. To discover that, according to the probabilistic interpretation, even a 'single' (indistinguishable) electron must be considered as a statistical entity, troubled even Einstein: hence his celebrated dismay over God's gambling habits. It seemed that God was playing a similar kind of dice-game with electrons to that which Einstein had been perfectly happy playing with colloids. The search for a way out of statistics continued: and continues to this day, in the form of such things as the 'hidden variable' theories of quantum mechanics. Not everyone, though, shared Einstein's disappointment, and the statistical interpretation of quantum mechanics seems actually to have been accepted if anything more readily than the original statistical ideas of kinetic theory. It seems reasonable to speculate then that the development and validation of classical statistical physics in the study of Brownian motion thus made quantum statistical mechanics rather easier to swallow: classical Brownian motion paved the way for the quantum revolution.

\section{Brownian motion and soft matter: to the present day}

In more recent years, the colloidal suspension (along with other forms of 'soft matter') has perhaps had its revenge for being so summarily dismissed in favour of the subatomic zoo in the early part of the last century. That the consequences of 'thermal forces' (i.e. Brownian motion) are most observable at the scale of colloidal suspensions is both the reason that colloids provided the original key to the establishment of statistical thermodynamics, and also why 'soft-matter' studies today are so important in modern 'mesotechnologies', such as foods, cosmetics, detergents, and biophysics. It is because of the viability of the organization of matter at this scale (through phase changes, metastable and non-equilibrium structure, and so on), under weak forces comparable to the thermal energy scale $k T$, that 'spontaneous' mesoscopic structure and function exist; that the modern world consists of and relies upon such things as plastics, detergents, paints, people, and cheese ${ }^{6}$.

Our understanding of the consequences of thermal forces in 'complex fluids' is of course far from complete, and this paper makes no attempt to consider the post-Perrin/Einstein developments of the theory of Brownian motion (see e.g. [25]). Experimentally, advances in optical technology and, especially, the invention of the laser have enabled a vast range of detailed, quantitative measurements: for instance the elaborate calculation of single-particle diffusion as in Henri's tracing of trajectories [16] may now be achieved by an almost routine dynamic light scattering measurement; Perrin's exhaustive particle sizing has certainly become a matter of routine in industrial 'particle sizers'; and the development of controlled methods

6 This list is not necessarily in order of importance or complexity. 
of fabrication of 'model' colloids has made the colloidal suspension an important tool in fundamental research across a wide range of disciplines [26]. The first decade of the last century was a beginning rather than an ending: even in relatively simple model colloidal suspensions, such features as interparticle and hydrodynamic interactions, glassy behaviour and 'crowded' environments, superlattice crystallinity in multi-size systems, effects of polydispersity and nonsphericity, and so on, still present enormous difficulties and enormous potential for advancing our understanding. Meanwhile 'soft matter' has of course gone beyond dilute suspensions of solid colloidal spheres, to embrace what we may term 'elemental types' such as surfactants, polymers, liquid crystals, and foams, not to mention mixtures of these. Grander goals, such as understanding the physics of biological systems and the 'complex' statistical phenomena underlying social, financial, and transport structures - these await us in the future. What is certain is that from little acorn-colloids first planted almost two hundred years ago, a forest of big soft trees continues to grow.

\section{Acknowledgments}

I would like to thank Wilson Poon for suggestions and discussion, and Jacques Leng for a critical reading of the manuscript.

\section{References}

[1] Nye M J 1972 Molecular Reality (London: Macdonald)

[2] Brush S G 2002 Am. J. Phys. 70119

[3] Gray S 1696 Phil. Trans. 19280

[4] Brown R 1828 Phil. Mag. 4171

[5] Brongniart A 1827 Ann. Sci. Naturelles 1241

[6] Gouy G 1888 J. Physique 7561

[7] Gouy G 1889 C. R. Acad. Sci., Paris 109102

[8] Gouy G 1895 Rev. Gén. Sci. 61

[9] Ostwald W 1895 Rev. Gén. Sci. 6956

[10] Einstein A 1905 Ann. Phys., Paris 17549

[11] Furth R (ed) 1926 Albert Einstein: Investigations on the Theory of the Brownian Movement (New York: Dover)

[12] Bachelier L 1900 Ann. Ecole Normale Supérieure 1721

[13] Smoluchowski M 1906 Ann. Phys., Paris 21772

[14] Langevin P 1908 C. R. Acad. Sci., Paris 146530

[15] Svedberg T 1906 Z. Elektrochem. 12853

[16] Henri V 1908 C. R. Acad. Sci., Paris 1461024

[17] Perrin J 1909 Ann. Chim. Phys. 181

[18] Cotton A 1908 Rev. Mois 5739

[19] Weeks E R, Crocker J C, Levitt A C, Schofield A and Weitz D A 2000 Science 287627

[20] Perrin J 1908 C. R. Acad. Sci., Paris 146967

[21] Perrin J 1908 C. R. Acad. Sci., Paris 147475

Perrin J 1908 C. R. Acad. Sci., Paris 147530

Perrin J 1908 C. R. Acad. Sci., Paris 147594

[22] Perrin J 1909 C. R. Acad. Sci., Paris 149349

Perrin J 1909 C. R. Acad. Sci., Paris 149477

[23] Perrin J 1911 C. R. Acad. Sci., Paris 1521165

Perrin J 1911 C. R. Acad. Sci., Paris 1521380

[24] Perrin J 1923 Atoms (London: Constable) (Engl. transl. D L Hammick)

[25] Kubo 1986 Science 233330

[26] Pusey P N 1989 Liquids, Freezing and Glass Transition ed J-P Hansen, D Levesque and J Zinn-Justin (Amsterdam: Elsevier) 